\title{
Is there an enhancement of photon strength at low $\gamma$-ray energies in Mo isotopes?
}

\author{
M. Krtička ${ }^{* a}$, U. Agvaanluvsan ${ }^{b}$, J.A. Becker ${ }^{b}$, F. Bečváŕ ${ }^{a}$, T.A. Bredeweg ${ }^{c}$, \\ R. Haight ${ }^{c}$, G.E. Mitchell ${ }^{d, e}$, J.M. O'Donnell ${ }^{c}$, W. Parker ${ }^{b}$, R. Reifarth ${ }^{c}$, \\ R.S. Rundberg ${ }^{c}$, E.I. Sharapov ${ }^{f}$, S. Sheets ${ }^{b, d}$, I. Tomandl ${ }^{g}$, J.L. Ullmann ${ }^{c}$, D. Vieira ${ }^{c}$, \\ J.M. Wouters ${ }^{c}$, J.B. Wilhelmy ${ }^{c}$, C.Y. Wu ${ }^{b}$ \\ ${ }^{a}$ Faculty of Mathematics and Physics, Charles University in Prague, \\ V Holešovičkách 2, CZ-180 00 Prague 8, Czech Republic \\ ${ }^{b}$ Lawrence Livermore National Laboratory, Livermore, California 94550, USA \\ ${ }^{c}$ Los Alamos National Laboratory, Los Alamos, New Mexico 87545, USA \\ ${ }^{d}$ North Carolina State University, Raleigh, North Carolina 27708, USA \\ e Triangle Universities Nuclear Laboratory, Durham, North Carolina 27708, USA \\ $f$ Joint Institute for Nuclear Research, 141980 Dubna, Russia \\ ${ }^{g}$ Nuclear Physics Institute, Czech Academy of Sciences, CZ-250 68 Řež, Czech Republic \\ E-mail: krticka@ipnp.troja.mff.cuni.cz
}

\begin{abstract}
A strong enhancement of the photon strength functions of the Mo isotopes has been recently reported from $\left({ }^{3} \mathrm{He}^{3} \mathrm{He}^{\prime} \gamma\right)$ and $\left({ }^{3} \mathrm{He}, \alpha \gamma\right)$ reactions [2]. In order to verify this interesting phenomenon a dedicated sum-coincidence measurement of the spectra of two-step $\gamma$ cascades following neutron capture in ${ }^{95}$ Mo was undertaken at the thermal neutron beam of the LWR-15 research reactor at ̌̌ež. Analysis of these spectra indicates a much weaker enhancement than reported in the ${ }^{3} \mathrm{He}$-induced reaction reaction measurements This conclusion is supported by a preliminary analysis of data from the ${ }^{95} \mathrm{Mo}(\mathrm{n}, \gamma){ }^{96} \mathrm{Mo}$ reaction at isolated $s-$ and $p$-wave neutron resonances. These data are from measurements with the DANCE $4 \pi$ detector array at the Los Alamos pulsed spallation neutron source facility.
\end{abstract}

Workshop on Photon Strength Functions and Related Topics

June 17-20 2007

Prague, Czech Republic

* Speaker. 


\section{Introduction}

Recently, a very strong enhancement of the radiative transition rate at low $\gamma$-ray energies has been reported in ${ }^{93-98} \mathrm{Mo}$ and ${ }^{56,57} \mathrm{Fe}$ isotopes $[1,2,3]$. To explain this enhancement a term that displays a tendency to diverge as $E_{\gamma} \rightarrow 0$ was suggested to be present in the expression for one of the photon strength functions (PSFs) responsible for the noted enhancement, specifically for the $E 1$, $M 1$ or $E 2$ photon strength functions; this interpretation arose from detailed analysis of the spectra of $\gamma$ rays produced from $\left({ }^{3} \mathrm{He}^{3} \mathrm{He}^{\prime} \gamma\right)$ and $\left({ }^{3} \mathrm{He}, \alpha \gamma\right)$ reactions at various ejectile energies [1, 2]. In the spirit of the Brink hypothesis [4] the enhancement can be accounted for as a manifestation of a vibrational mode that is characteristic by an extremely low energy and is coupled to each of the nuclear levels. In accordance with the nomenclature introduced in Refs. [1, 2], this putative mode will be referred to as a softpole mode. For ${ }^{57} \mathrm{Fe}$ this conclusion has been supported by data on TwoStep $\gamma$ Cascades (TSCs) accompanying the capture of thermal neutrons in ${ }^{56} \mathrm{Fe}$, see Refs. $[1,3]$. The existence of a softpole mode may have a significant impact on the present understanding of photon production associated with the decay of highly excited nuclear levels.

One crucial question is whether the observed effect in ${ }^{3} \mathrm{He}$-induced reactions is a manifestation of a $\gamma$ emission from product nuclei prior to reaching complete thermalization. It can be argued that the data on TSCs in ${ }^{57} \mathrm{Fe}$ refute this explanation, as the emission of $\gamma$ rays following any slowneutron capture reaction is almost exclusively a compound-nucleus process. However, following our recent GEANT3 simulations of a similar TSC experiment [5], it appears that the spectra of TSCs in ${ }^{57} \mathrm{Fe}$ are contaminated by the soft brehmsstrahlung induced by extremely strong primary transitions to the ground state and to the $14 \mathrm{keV}$ level of ${ }^{57} \mathrm{Fe}$. This brehmsstrahlung may mimic the effect of a strong enhancement of primary transitions with energies $E_{\gamma} \lesssim 2 \mathrm{MeV}$. In addition, the relatively low level density of the ${ }^{57} \mathrm{Fe}$ nucleus is expected to lead to strong fluctuations in the TSC spectra that might also mimic the enhancement at low $\gamma$-ray energies. These considerations weaken the conclusion that the softpole term is necessary for interpreting TSC data [6].

It should be noted that the method for analyzing the data from ${ }^{3} \mathrm{He}$-induced reactions has not yet been subject to stringent validation tests. In view of this, one cannot completely rule out the possibility that the observed enhancement of soft radiative transitions is an artifact of the analysis method itself.

These arguments motivated us to undertake experimental studies of (i) TSCs following thermal neutron capture in ${ }^{95} \mathrm{Mo}$, and (ii) singles spectra for different multiplicities from isolated neutron resonances in ${ }^{96} \mathrm{Mo}$ measured with the DANCE $4 \pi \mathrm{BaF}_{2}$ detector array. Descriptions of the experimental setups of both experiments are given in Section 2. Gamma-ray spectra obtained from these studies are compared with simulations based on the extreme statistical model of the nucleus using the DICEBOX code. The simulations are described in Section 3 and compared to experimental data in Section 4. A discussion of results and a summary is given in the last section.

\section{Experiments}

\subsection{TSC experiment}

The TSC measurement was performed at the 15 MW light-water reactor at Řež. As a full 


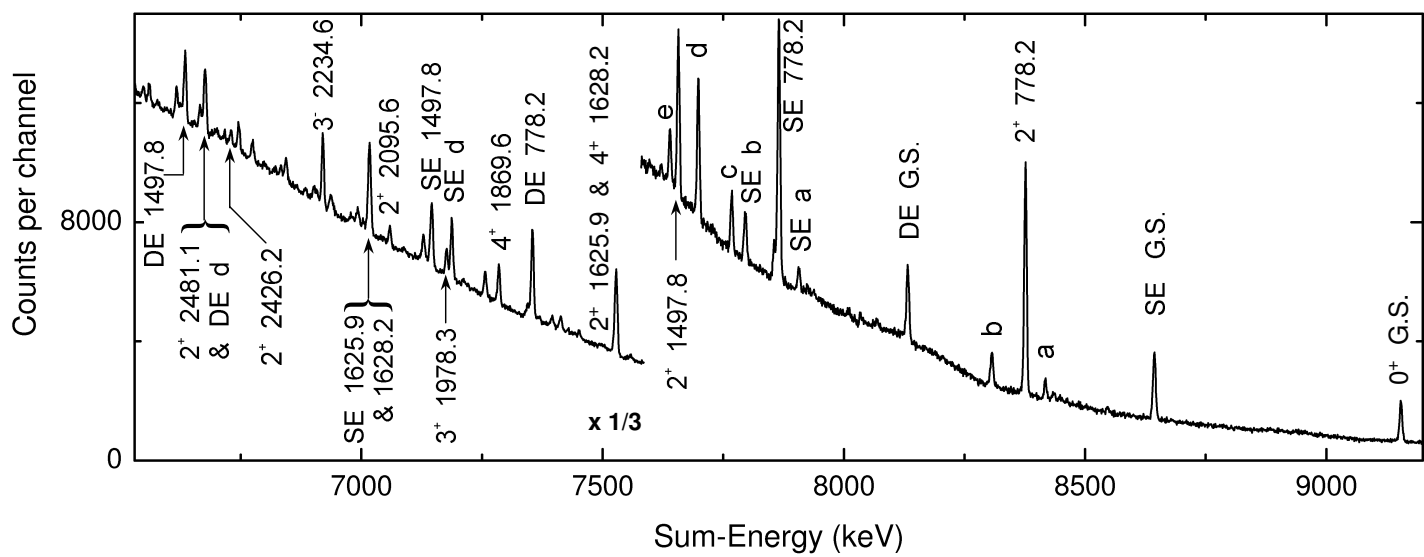

Figure 1: The spectrum of $\gamma$-ray energy sums accumulated from the event-mode data obtained from the coincidence neutron capture measurement using a natural Mo sample. The lines with labels a, b, c, ... originate from the full energy deposition of two non-contiguous steps of $\gamma$ cascades; energies of the transitions involved are listed in Table 1.

description of the sum-coincidence experimental setup and the TSC technique used is given elsewhere [7], here we give only the details specific to the present measurement.

A very pure beam of thermal neutrons, collimated to a cross section $2.5 \times 0.25 \mathrm{~cm}^{2}$ at the sample position, had a flux of about $3 \times 10^{6} \mathrm{n} \mathrm{cm}^{-2} \mathrm{~s}^{-1}$. Neutron capture $\gamma$-rays were detected by two HPGe detectors with $25 \%$ efficiency. They were placed on the oposite sides of the target at $90^{\circ}$ with respect to the beam and close to the sample to increase the coincidence rate. A natural molybdenum sample with a weight of about $6 \mathrm{~g}$ was used. Thanks to the combination of the relatively high abundance of ${ }^{95} \mathrm{Mo}$ and its large cross section, the background from other Mo isotopes was very small in the TSC measurement and is not visible in the measured spectra. Data on the deposited $\gamma$-ray energies and the detection time difference were recorded. The measurement lasted about 300 hours with an average counting rate of 150 coincidences/s.

The spectrum of $\gamma$-ray energy sums is shown in Fig. 1. The lines that are relevant for the present study originate from the full deposition of the energy carried by two contiguous $\gamma$-cascade steps on the condition that the first step initiates at the thermal neutron capturing state and the second step ends at one of the low-lying levels in ${ }^{57} \mathrm{Fe}$. These lines are labeled with the value of the energy and the $J^{\pi}$ of the corresponding low-lying level. Each such "full energy" (FE) line is accompanied by its single-escape (SE) and double-escape (DE) satellite lines; these are labeled in Fig. 1 with the level energy and either SE or DE. It should be stressed that the origin of all lines observed in the spectrum of $\gamma$-ray energy sums is well understood, but for the sake of clarity, in Fig. 1 only a fraction of these lines are labeled.

From the information recorded a set of spectra of $\gamma$-rays were obtained that belong to all TSCs that end at preselected final levels in ${ }^{96}$ Mo. Each of these spectra was constructed from the deposited energy in one of the detectors under the condition that the $\gamma$-ray energy sum from both detectors fell within the region of the FE line corresponding to a preselected level. The energies and $J^{\pi}$ of these final levels are listed in Table 2. Note, that in one case two of the levels were not resolved. In the following we call these spectra TSC spectra and the corresponding final levels - 
Table 1: List of non-contiguous pairs of $\gamma$-cascade steps that are responsible for parasitic lines and their SE and DE satellites in the spectrum of energy sums. The labeling used coincides with that in Fig. 1. Abbreviations "C.S." and "G.S." stand for the neutron capturing state and the ground state of the nucleus, respectively; energies of the intermediate levels are given in $\mathrm{keV}$.

\begin{tabular}{cccc}
\hline \hline Label & Transitions & Label & Transitions \\
\hline a & C.S. $\rightarrow 2235 \& 1497 \rightarrow$ G.S. & d & C.S. $\rightarrow 2235 \& 778 \rightarrow$ G.S. \\
b & C.S. $\rightarrow 1626 \& 778 \rightarrow$ G.S. & e & C.S. $\rightarrow 2235 \& 1497 \rightarrow 778 \mathrm{keV}$ \\
c & C.S. $\rightarrow 2235 \& 1626 \rightarrow 778 \mathrm{keV}$ & & \\
\hline \hline
\end{tabular}

Table 2: TSC final levels. Their labels, excitation energies and $J^{\pi}$ assignments are listed. Label 4 refers to a doublet of unresolved levels; their energies are listed in italics.

\begin{tabular}{ccccccccc}
\hline \hline Label & Energy $(\mathrm{keV})$ & $J^{\pi}$ & Label & Energy $(\mathrm{keV})$ & $J^{\pi}$ & Label & Energy $(\mathrm{keV})$ & $J^{\pi}$ \\
\hline 1 & 0.0 & $0^{+}$ & 6 & 1978.3 & $3^{+}$ & 10 & 2438.4 & $5^{+}$ \\
2 & 778.3 & $2^{+}$ & 7 & 2095.6 & $2^{+}$ & 11 & 2594.2 & $3^{+}$ \\
3 & 1497.8 & $2^{+}$ & 8 & 2234.6 & $3^{-}$ & 4 & 1625.9 & $2^{+}$ \\
5 & 1869.5 & $4^{+}$ & 9 & 2426.2 & $2^{+}$ & & 1628.2 & $4^{+}$ \\
\hline \hline
\end{tabular}

\section{TSC final levels.}

While constructing the TSC spectra, the background due to accidental coincidences and Compton scattering was subtracted: Compton background was subtracted by choosing background regions on the two sides of each peak in the spectrum of energy sums. Time windows, selecting three intervals of detection-time difference, were adjusted to isolate the net signal from the background due to accidental coincidences; for details see Ref. [7].

The constructed TSC spectra were corrected for the energy dependence of the FE line efficiencies of both detectors. In addition, corrections for the vetoing effect caused by the detection of $\gamma$-rays following the decay of the TSC final level and for the effects of $\gamma-\gamma$ angular correlations, see e.g. [8], were applied. After these corrections the TSC spectra were converted into spectra expressed in absolute TSC intensities. This conversion requires knowledge of the TSC intensity of at least one TSC and it was performed with data from other experiments [9].

An example of a TSC spectrum is given in Fig. 2. It demonstrates the large dynamic range of TSC intensities, as well as the symmetry around the midpoint of the spectrum. The energies of the intermediate levels responsible for some of the pairs of $\gamma$ lines are shown. The insert in Fig. 2 illustrates the presence of the TSCs proceeding via intermediate levels in the level quasicontinuum of ${ }^{96} \mathrm{Mo}$.

To ease the task of comparing the experimental TSC spectra with predictions resulting from various assumptions about the level density and the photon strength functions, the role of experimental errors as well as uncertainties due to residual Porter-Thomas fluctuations [10] in the behav- 


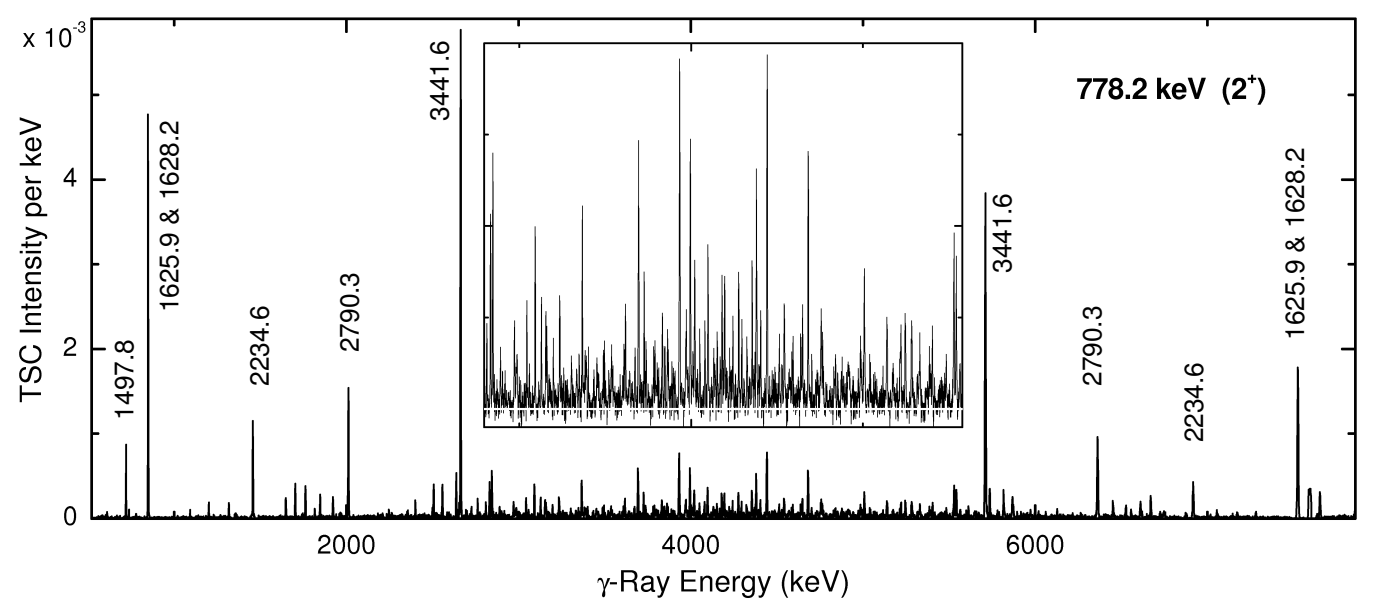

Figure 2: The spectrum of TSCs in ${ }^{96}$ Mo belonging to the $J^{\pi}=2^{+}$TSC final level at $778.2 \mathrm{keV}$ in ${ }^{96} \mathrm{Mo}$.

ior of the TSC spectra must be suppressed. To achieve this we transformed the experimental TSC spectra into a histogram representation characterized by coarse energy bins. In our case we chose bin widths of $100 \mathrm{keV}$. The TSC spectra transformed in this way are referred to as the wide-bin TSC spectra. For the same reason, each of the TSC spectra has been integrated over the energy interval centered with respect to the midpoints of the spectrum. In this manner we obtained what we call the integrated TSC intensities. Together with their experimental uncertainties they were determined from integration over an interval with a width of $\Delta E_{\gamma}=4 \mathrm{MeV}$.

\subsection{DANCE experiment}

Measurement of singles $\gamma$-ray spectra for different multiplicities following neutron capture at isolated resonances was performed at the spallation neutron source of the Los Alamos Neutron Science Center LANSCE [11]. The 800-MeV H${ }^{-}$beam of about $625 \mu$ s duration from the LANSCE linac is converted to protons by thin foil stripping and injected into the proton storage ring. The injected beam is stacked on itself until protons from the entire linac macropulse are stored. This reduces the proton pulse width to about $125 \mathrm{~ns}$. This pulse is extracted with a repetition rate of 20 $\mathrm{Hz}$ and transported to a tungsten spallation target, where fast neutrons are created. After passing through a water moderator, the neutrons with a white energy spectrum enter evacuated flight paths. The DANCE detector array is installed on the 20m neutron flight path 14 [11].

The DANCE multiplicity spectrometer [12] is $\mathrm{a} \simeq 4 \pi \mathrm{BaF}_{2}$ crystal array designed for studying neutron capture cross sections on small quantities of radioactive material or isotopic samples. The DANCE array consists of $160 \mathrm{BaF}_{2}$ scintillation crystals, which detect $\gamma$-rays following a neutron capture. Each crystal has a length of $15 \mathrm{~cm}$, forming an inner radius of $17 \mathrm{~cm}$ of the ball. Neutrons scattered into the detector can be captured on the barium isotopes and produce an undesirable background. To reduce this background, a ${ }^{6} \mathrm{LiH}$ shell of about $4 \mathrm{~cm}$ thick is placed between the sample and the $\mathrm{BaF}_{2}$ crystals. A molybdenum foil with a thickness of $25 \mathrm{mg} / \mathrm{cm}^{2}$ and enrichment of $96.47 \%$ in ${ }^{95} \mathrm{Mo}$ was used in the experiment that lasted about one week.

The DANCE acquisition system [13] is based on waveform digitization for all $160 \mathrm{BaF}_{2}$ detectors. Our interest focused on singles spectra for different multiplicities. Because of the relatively 
small size of each crystal, there is a strong cross talk between neighboring crystals in their response to a single photon. Therefore contiguous crystals, that have fired during a restricted time interval, are combined into one single "cluster".

The cluster $\gamma$-ray multiplicity spectra are obtained for many neutron resonances under the constraint that the detected energy sum, $E_{\Sigma}$, is close to the Q-value of the reaction. Specifically, only events with detected energy sum $E_{\Sigma}=7.8-9.3 \mathrm{MeV}$ were considered. Note in this context that the neutron separation energy for ${ }^{96} \mathrm{Mo}$ is $9.154 \mathrm{MeV}$. The multiplicity distribution, together with the shapes of singles spectra for individual multiplicities, enabled us to determine the spin and parity of strong resonances [14].

In order to suppress experimental errors, as well as the role of Porter-Thomas fluctuations, in simulations the spectra were binned into coarse bins with a width of $200 \mathrm{keV}$, similar to the wide-bin TSC spectra.

\section{Numerical Simulations of the $\gamma$-Decay of the Compound Nucleus ${ }^{96}$ Mo}

For the simulation of $\gamma$ decay following neutron capture, the code DICEBOX [15] was used. In this code the level scheme of the nucleus and the associated decay are artificially generated according to an adopted level density model and assumed models of the PSFs. However, below some critical energy - in the present case below $2.8 \mathrm{MeV}$ - the level energies, spins, parities and $\gamma$-branching ratios are taken from the literature [16]. Hereafter the generated level structure and the decay scheme are called a nuclear realization. While the level structure below the critical energy is kept fixed, many nuclear realizations are generated in a simulation run. For each nuclear realization many $\gamma$ cascades, initiating at the neutron capturing state and terminating at the ground state, are randomly generated following the rules of the extreme statistical model. Thanks to the introduction of the technique of precursors [15], the code offers the unique feature of rigorous simulation of the residual Porter-Thomas fluctuations of any cascade-related quantity. In our case, the quantities of interest are the wide-bin and integrated TSC spectra from thermal neutron capture and single spectra for different multiplicities from resonances of given spin and parity. Of course, the detector response to simulated cascades must be applied prior to comparison of simulated spectra to those measured at DANCE. Simulations of the detector response were performed using GEANT 3 package. We typically simulated 50000 events in each of the 50 nuclear realizations in the case of TSCs and 20000 events in 20 realizations for the resonance capture. The smaller numbers for the latter case are required by the time-consuming GEANT 3 simulations.

\subsection{Photon strength functions tested}

Our primary interest in analyzing TSC data on ${ }^{96} \mathrm{Mo}$ was focused on testing the validity of the main conclusion from the ${ }^{96} \mathrm{Mo}\left({ }^{3} \mathrm{He},{ }^{3} \mathrm{He}^{\prime}, \gamma\right){ }^{96} \mathrm{Mo}$ and ${ }^{97} \mathrm{Mo}\left({ }^{3} \mathrm{He}, \alpha \gamma\right){ }^{96} \mathrm{Mo}$ data [2]. Specifically, that the expressions for the mixed PSF, represented by the function $f\left(E_{\gamma}\right)$ according to Eq. (3.4), see below, involve a term displaying a singularity at $E_{\gamma}=0$, see Sec. 3.1.3. However, in an effort to achieve the best possible agreement between the predicted cascade-related observables and their experimental values we also tested, in the spirit of the trial-and-error approach, a large number of ad hoc postulated expressions for the PSFs. 
In an initial phase of this approach we used the $E 1$ PSFs according to the Axel-Brink (AB) [4, 17], KMF [18] or GLO [19] models, as described in Sec. 3.1.1, while in the case of $M 1$ we started with the single-particle (SP) model [20], spin-flip (SF) model [21] and scissors-resonance (SR) model, see Sec. 3.1.2.

\subsection{1 $E 1$ photon strength functions}

The $E 1$ PSF according to the "standard" BA model is given by the Lorentzian form [4, 17]:

$$
f_{E 1}^{(\mathrm{BA})}\left(E_{\gamma}\right)=\frac{1}{3(\pi \hbar c)^{2}} \cdot \frac{\sigma_{\mathrm{G}} E_{\gamma} \Gamma_{\mathrm{G}}^{2}}{\left(E_{\gamma}^{2}-E_{\mathrm{G}}^{2}\right)^{2}+E_{\gamma}^{2} \Gamma_{\mathrm{G}}^{2}} .
$$

Here, $E_{\mathrm{G}}, \Gamma_{\mathrm{G}}$ and $\sigma_{\mathrm{G}}$ are the energy, damping width and maximum of the photonuclear cross section. Relying on photonuclear data for ${ }^{96} \mathrm{Mo}$ [22], we adopted $E_{\mathrm{G}}=16.20 \mathrm{MeV}, \Gamma_{\mathrm{G}}=6.01$ $\mathrm{MeV}$, and $\sigma_{\mathrm{G}}=185 \mathrm{mb}$. We arrived at these values from fitting the cross sections in an energy region from 11 to $20 \mathrm{MeV}$.

Two upgraded models for the $E 1$ PSF that we tested are characterized by a partial violation of Brink's hypothesis. In these models the GDER changes in shape and size with the nuclear temperature $T$ of the level on which it is built. Of these models, the KMF model proposed in Ref. [18], is not phenomenological. It results from microscopic calculations within the framework of the semi-microscopic shell-model approach, which is based on the results of Fermi liquid theory. This model was suggested as an approximation describing the behavior of the E1 PSF at the lowenergy tail of the electric GDER of spherical nuclei. The expression for the $E 1$ PSF is

$$
f_{E 1}^{(\mathrm{KMF})}\left(E_{\gamma}, T\right)=\frac{0.7}{3(\pi \hbar c)^{2}} \cdot \frac{\sigma_{\mathrm{G}} E_{\mathrm{G}} \Gamma_{\mathrm{G}} \Gamma\left(E_{\gamma}, T\right)}{\left(E_{\gamma}^{2}-E_{\mathrm{G}}^{2}\right)^{2}}, \quad \Gamma\left(E_{\gamma}, T\right)=\Gamma_{\mathrm{G}} \frac{E_{\gamma}^{2}+4 \pi^{2} T^{2}}{E_{\mathrm{G}}^{2}},
$$

where $T=T(E) \equiv \sqrt{(E-\Delta) / a}$, with $E, \Delta$ and $a$ being excitation energy of a final level, pairing energy and the shell-model level-density parameter, respectively.

We also tested the semi-empirical model GLO developed for spherical or weakly deformed nuclei, see Ref. [19]. This model predicts that

$$
f_{E 1}^{(\mathrm{GLO})}\left(E_{\gamma}, T\right)=\frac{\sigma_{\mathrm{G}} \Gamma_{\mathrm{G}}}{3(\pi \hbar c)^{2}}\left[\frac{E_{\gamma} \Gamma\left(E_{\gamma}, T\right)}{\left(E_{\gamma}^{2}-E_{\mathrm{G}}^{2}\right)^{2}+E_{\gamma}^{2} \Gamma\left(E_{\gamma}, T\right)^{2}}+\frac{4 \pi^{2} F_{K} \Gamma_{\mathrm{G}} T^{2}}{E_{\mathrm{G}}^{5}}\right] .
$$

The same values of parameters $E_{\mathrm{G}}, \Gamma_{\mathrm{G}}, \sigma_{\mathrm{G}}$ as in the case of BA model were used in the KMF and GLO models. Compared to the KMF model, the GLO model does not display the divergence of the $E 1$ PSF for $E_{\gamma} \rightarrow E_{\mathrm{G}}$.

\subsection{2 $M 1$ and $E 2$ photon strength functions}

The $M 1$ PSF according to the SP model is $\gamma$-ray energy independent, $f_{M 1}^{(\mathrm{SP})}=$ const., see Ref. [20], while in the cases of SF and SR models the explicit expressions for $f_{M 1}^{(\mathrm{SF})}$ and $f_{M 1}^{(\mathrm{SR})}$ are given by the right-hand side of Eq. (3.1) with the set of parameters $\left\{E_{\mathrm{G}}, \Gamma_{\mathrm{G}}, \sigma_{\mathrm{G}}\right\}$ replaced by sets $\left\{E_{\mathrm{SF}}, \Gamma_{\mathrm{SF}}, \sigma_{\mathrm{SF}}\right\}$ and $\left\{E_{\mathrm{SR}}, \Gamma_{\mathrm{SR}}, \sigma_{\mathrm{SR}}\right\}$, respectively.

Following the data in Ref. [19] the parameters of the spin-flip model were adjusted to the values $E_{\mathrm{SF}}=8.95 \mathrm{MeV}$ and $\Gamma_{\mathrm{SF}}=4 \mathrm{MeV}$. The remaining parameter, the peak photoabsorption 
cross section of the spin-flip resonance $\sigma_{\mathrm{SF}}$, was adjusted to reproduce the ratio $f_{E 1} / f_{M 1}$ at $E_{\gamma} \approx$ $7 \mathrm{MeV}$; this is about 7 for $A \gtrsim 90$ nuclei [23]. With the explicit expression for $E 1$ PSF, this ratio makes it possible to determine $\sigma_{\mathrm{SF}}$. The same approach was also applied for determining the constant value of $f_{\mathrm{M} 1}$ in the $M 1$ SP model.

Data from nuclear resonance fluorescence experiments on ${ }^{94}$ Mo [24] and ${ }^{96}$ Mo [25] yield a total reduced $B(M 1) \uparrow$ strength from the ground state to $1^{+}$states in the energy region $2.5 \mathrm{MeV} \lesssim$ $E_{\gamma} \lesssim 3.5 \mathrm{MeV}$ represented by $\sum B(M 1) \uparrow \approx 0.5 \mu_{N}^{2}$. This is about one order of magnitude smaller compared to the strength observed in well-deformed rare-earth nuclei. This indicates a small role of the scissors mode in the $\gamma$ decay of nuclei with $A \simeq 95$, which is consistent with their low value of deformation parameter, $\delta \approx 0.16$. Assuming that $\Gamma_{\mathrm{SR}} \approx 0.6 \mathrm{MeV}$ and that the total reduced $B(M 1) \uparrow$ strength for ${ }^{96} \mathrm{Mo}$ amounts to $\approx 0.5 \mu_{N}^{2}$, one expects a peak cross section value $\sigma_{\mathrm{SR}} \approx 0.1 \mathrm{mb}$.

For the $E 2 \mathrm{PSF}$ the single particle model was used in the calculations with the value $f_{E 2}^{(\mathrm{SP})}=$ $5 \times 10^{-11} \mathrm{MeV}^{-5}$.

\subsubsection{The mixed PSF following from the $\left({ }^{3} \mathrm{He}^{3}{ }^{3} \mathrm{He}^{\prime} \gamma\right)$ and $\left({ }^{3} \mathrm{He}, \alpha \gamma\right)$ reactions}

In order to test the enhancement of the photon strength at low $\gamma$-ray energies observed in ${ }^{96} \mathrm{Mo}\left({ }^{3} \mathrm{He},{ }^{3} \mathrm{He}^{\prime} \gamma\right){ }^{96} \mathrm{Mo}$ and ${ }^{97} \mathrm{Mo}\left({ }^{3} \mathrm{He}, \alpha \gamma\right){ }^{96} \mathrm{Mo}$ reactions we performed - as our main task simulations with a mixed PSF given according to Refs. [1,2] by

$$
f\left(E_{\gamma}, T\right)=\kappa\left[f_{E 1}^{(\mathrm{KMF})}\left(E_{\gamma}, T\right)+f_{M 1}^{(\mathrm{SF})}\left(E_{\gamma}\right)+f^{(\mathrm{pole})}\left(E_{\gamma}\right)\right]+E_{\gamma}^{2} f_{E 2}
$$

with an enhancement term

$$
f^{(\text {pole })}\left(E_{\gamma}\right)=\frac{A}{3(\pi \hbar c)^{2}} E_{\gamma}^{-b},
$$

where, $\kappa, A$ and $b$ are free parameters.

In accordance with what was deduced from the ${ }^{97} \mathrm{Mo}\left({ }^{3} \mathrm{He}, \alpha \gamma\right){ }^{96} \mathrm{Mo}$ data in the $\gamma$-ray energy region above $1 \mathrm{MeV}$, see Ref. [2], we chose $A=0.47 \mathrm{mb} / \mathrm{MeV}, b=2.7$ and $\kappa=0.32$. Unlike Ref. [2], in the spirit of the KMF model we took into account the temperature dependence of the E1 PSF.

As already mentioned, the $\left({ }^{3} \mathrm{He}, \alpha \gamma\right)$ and $\left({ }^{3} \mathrm{He}^{3}{ }^{3} \mathrm{He}^{\prime} \gamma\right)$ data yield information on the quantity $f\left(E_{\gamma}\right)$, but not about its multipolarity composition. This implies that in principle the term, $f^{(\text {pole })}\left(E_{\gamma}\right)$ may be assigned to any multipolarity.

Experimental data on the mixed PSF from $\left({ }^{3} \mathrm{He},{ }^{3} \mathrm{He}^{\prime} \gamma\right)$ and $\left({ }^{3} \mathrm{He}, \alpha \gamma\right)$ reactions are available only for energies $E_{\gamma} \gtrsim 1 \mathrm{MeV}$. For this reason in our simulations we considered also a modified enhancement term, called hereafter a restricted softpole term (RP). Specifically, we approximated the shape of PSFs at low $E_{\gamma}$ with two additional low-lying resonances (LLRs) of Lorentzian shape. The shapes corresponding to PSFs involving $f^{(\text {pole })}\left(E_{\gamma}\right)$ and $f^{(\mathrm{RP})}\left(E_{\gamma}\right)$ terms are shown in Fig. 3.

\subsection{Nuclear Level Densities}

We used almost exclusively the back-shifted Fermi Gas (BSFG) model [26]:

$$
\rho(E, J, \pi)=f(J) f(\pi) \frac{e^{2 \sqrt{a\left(E-E_{1}\right)}}}{12 \cdot 2^{1 / 2} \sigma_{c} a^{1 / 4}\left(E-E_{1}\right)^{5 / 4}},
$$


where $a$ and $E_{1}$ are adjustable parameters, while

$$
f(J)=\exp \left(\frac{-J^{2}}{2 \sigma_{c}^{2}}\right)-\exp \left(\frac{-(J+1)^{2}}{2 \sigma_{c}^{2}}\right) \quad \text { and } \quad \sigma_{c}^{2}=0.0888 A^{2 / 3} \sqrt{a\left(E-E_{1}\right)}
$$

are expressions for the spin probability distribution function $f(J)$ and spin cut-off parameter $\sigma_{c}$.

The level density at high excitation energies is expected to be parity independent, which corresponds to $f(\pi)=0.5$ in Eq. (3.7). On the other hand, the parity dependence at low energies remains in question. Level schemes from other experiments [16] show significant parity asymmetry below about $3 \mathrm{MeV}$. Therefore we used the formula from Ref. [27]

$$
f(\pi=+)=\frac{1}{2}\left(1+\frac{1}{1+\exp \left(C_{\pi}\left(E-\Delta_{\pi}\right)\right)}\right),
$$

where $\Delta_{\pi}$ is, roughly speaking, the excitation energy at which the parity-dependent level density changes to parity-independent, with the rate given by $C_{\pi}$. The negative-parity level density is $f(\pi)$, which is given by $f(\pi=-)=1-f(\pi=+)$.

To characterize the level density in ${ }^{96}$ Mo we followed the systematics in Ref. [26] and chose $a=10.19 \mathrm{MeV}^{-1}$ and $E_{1}=0.71 \mathrm{MeV}$. Parameters characterizing the parity-dependence of the level density were set to $\Delta_{\pi}=3.2 \mathrm{MeV}$ and $C_{\pi}=1.0 \mathrm{MeV}^{-1}$. The level density obtained from the Oslo measurements [28] is in agreement with this model.

\section{Results}

\subsection{Testing the presence of a strong softpole in a mixed photon strength function}

Data on the mixed PSF deduced from the ${ }^{96} \mathrm{Mo}\left({ }^{3} \mathrm{He},{ }^{3} \mathrm{He}^{\prime} \gamma\right){ }^{96} \mathrm{Mo}$ and ${ }^{97} \mathrm{Mo}\left({ }^{3} \mathrm{He}, \alpha \gamma\right){ }^{96} \mathrm{Mo}$ reactions [2] are plotted in the left panel of Fig. 3. If the models for the PSFs involved in the mixed PSF according to Eqs. $(3.4,3.5)$ are correct, the data points from the ${ }^{96} \mathrm{Mo}\left({ }^{3} \mathrm{He},{ }^{3} \mathrm{He}^{\prime} \gamma\right){ }^{96} \mathrm{Mo}$ and ${ }^{97} \mathrm{Mo}\left({ }^{3} \mathrm{He}, \alpha \gamma\right){ }^{96} \mathrm{Mo}$ reactions in Fig. 3 must be situated within the region limited by a pair of smooth curves. The lower and upper curve is given by Eq. (3.4) choosing, respectively, $T=0$ and $T=\sqrt{\left(B_{\mathrm{n}}-E_{\gamma}\right) / a}$. As can be seen, within the experimental errors the data points behave as expected.

Figure 3 illustrates the integrated TSC intensities and the results of the corresponding simulations, which assume the validity of the two adopted expressions for the mixed PSF. The data from the experiment and the results from the simulations are shown for 11 TSC spectra with labeling identical to that in Table 2. The experimental errors of the measured TSC intensities, as well as the rms values of the residual Porter-Thomas uncertainties of the simulated intensities, are indicated. As seen from the legend in Fig. 3, both the $E 1$ and $M 1$ origin of the enhancement terms $f^{(\text {pole })}\left(E_{\gamma}\right)$ and $f^{(\mathrm{RP})}\left(E_{\gamma}\right)$ were assumed while simulating the integrated TSC intensities.

Inspection of the results in Fig. 3 leads to the unambiguous conclusion that the mixed PSF given by Eqs. $(3.4,3.5)$ is in sharp disagreement with our experimental data on integrated TSC intensities. This disagreement remains even when the enhancement term $f^{(\text {pole })}\left(E_{\gamma}\right)$ is replaced by $f^{(\mathrm{RP})}\left(E_{\gamma}\right)$. These conclusions hold regardless the multipolarity ascribed to the enhancement terms $f^{\text {(pole) }}\left(E_{\gamma}\right)$ and $f^{(\mathrm{RP})}\left(E_{\gamma}\right)$. An illustration of the disagreement between experiment and simulations under the assumption that the $\gamma$ emission is governed by the mixed PSF [2] with the $M 1$ origin 

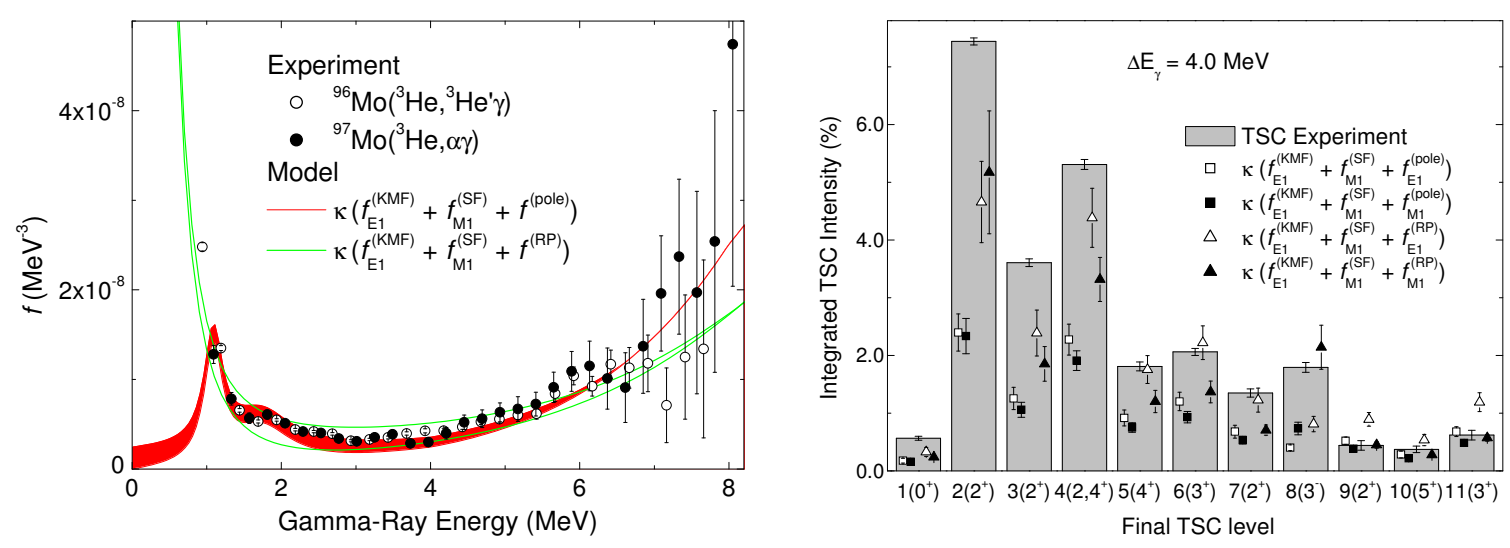

Figure 3: Lines and the shaded area in the left panel - the range of predictions for $f\left(E_{\gamma}, T\right)$ using terms $f^{(\text {pole })}$ and $f^{(\mathrm{RP})}$, respectively. Columns and points in the right panel - the integrated TSC intensities and the outcome of their DICEBOX simulations.

of the enhancement term $f^{(\text {pole })}\left(E_{\gamma}\right)$ for wide-bin TSC spectra is given on the left side of Fig. 4. The wide-bin TSC data are represented by points with error bars. Predictions of TSC spectra from simulations together with the associated residual Porter-Thomas uncertainties are represented by gray areas. In each energy bin the vertical range of the gray area is equal to two rms deviations of the TSC intensity, as deduced from a set of simulations for a large number of nuclear realizations. The expectation value of the TSC intensity is represented by the midpoint of the vertical range.

In Fig. 5 experimental singles spectra for different multiplicities corresponding to decay of $2^{+}$ and $3^{-}$resonances are compared to results from the preliminary simulations based on the model involving the $f_{M 1}^{(\mathrm{RP})}$ term. The agreement between experiment and simulation is again not very good. A general trend is the overprediction of events in the spectra for higher multiplicities. This is a consequence of the enhancement of a PSF at low $E_{\gamma}$ 's.

\subsection{A search for the best estimate of photon strength functions by the trial-and-error method}

With the trial -and-error approach we undertook more than 200 independent DICEBOX [15] simulations of the TSC spectra and more than 20 simulations of decay of isolated resonances for various postulated PSFs. The smaller number of simulations in the latter case was determined by the fact that GEANT3 simulations of detector response are rather time consuming. We started with testing of "standard" models of PSFs that were described in Sec. 3. None were able to account for the behavior of the cascade-related quantities. The rest of the simulations were done with some modifications of the standard PSFs. We shall not list the results of the whole set of simulations, but present only a summary of the most important results.

Very good agreement between the simulations and the TSC data was achieved with the empirical expressions for PSFs as follows.

For $E 1$ PSFs we used

$$
f_{E 1}^{(\text {emp.1) }}\left(E_{\gamma}, T\right)=f_{E 1}^{(\mathrm{KMF})}\left(E_{\gamma}, T\right) \times\left\{\begin{array}{cllr}
\lambda & \text { for } & E_{\gamma} \leq E_{\gamma 1} \\
Q\left(E_{\gamma}\right) & \text { for } & E_{\gamma 1}<E_{\gamma} \leq E_{\gamma 2} \\
1 & \text { for } & E_{\gamma}>E_{\gamma 2}
\end{array}\right.
$$



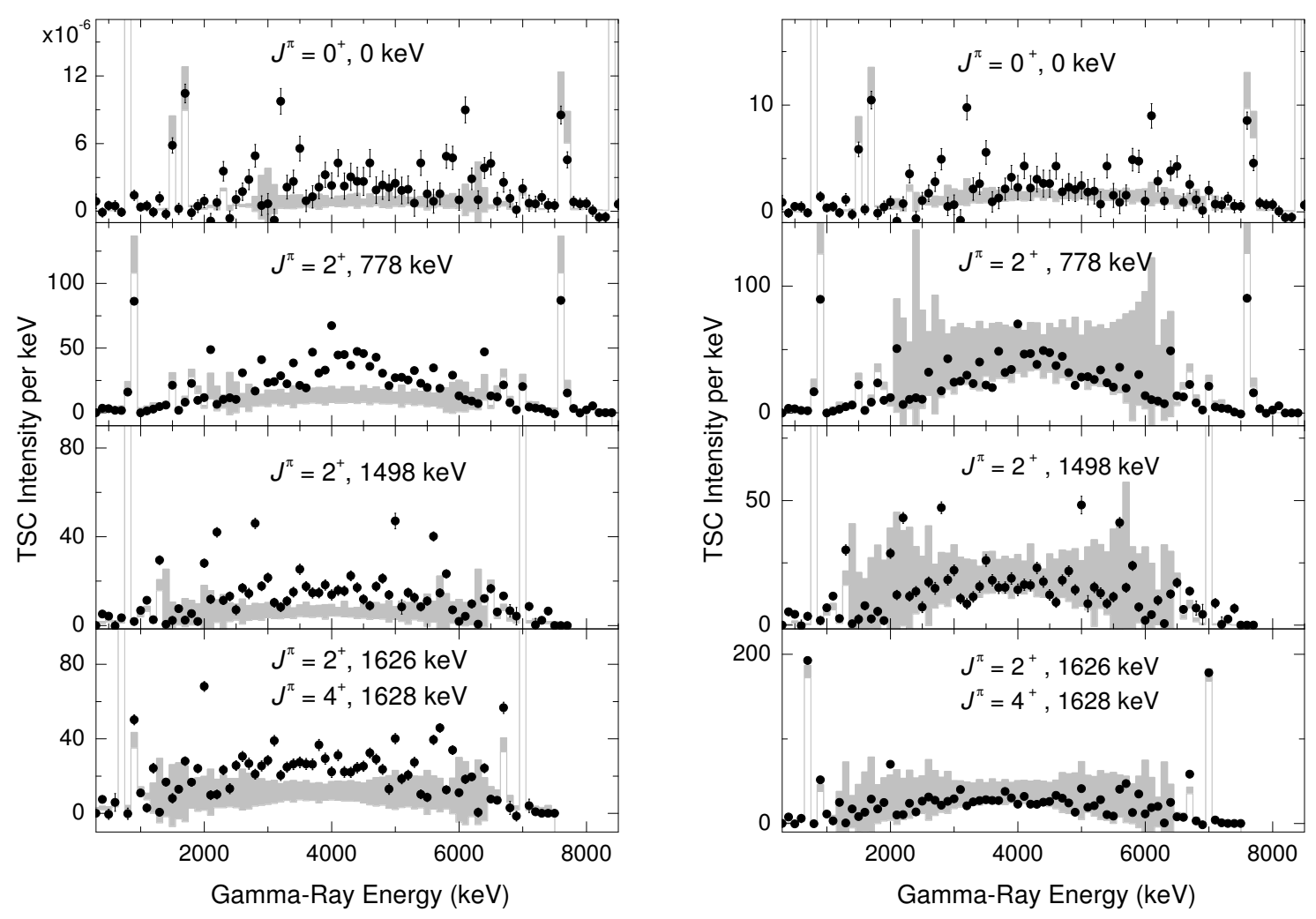

Figure 4: Comparison of experimental data (points) with simulations for wide-bin TSC spectra. The simulated spectra are represented by the gray areas, see the main text. The models used for simulations are those given by Eqs (3.4) and (3.5) (left panel) and by Eqs. (4.1) and (4.3) (right panel).

with

$$
Q\left(E_{\gamma}\right)=\lambda+(1-\lambda) \frac{E_{\gamma}-E_{\gamma 1}}{E_{\gamma 2}-E_{\gamma 1}} .
$$

The parameters involved were adjusted to: $\lambda=0.5, E_{\gamma 1}=4.0 \mathrm{MeV}$ and $E_{\gamma_{2}}=8.0 \mathrm{MeV}$.

The optimum choice of $M 1$ PSF we arrived at is represented by a sum of three resonance terms

$$
f_{M 1}^{(\mathrm{emp} .1)}\left(E_{\gamma}\right)=f_{M 1}^{(\mathrm{SF})}\left(E_{\gamma}\right)+f_{M 1}^{(\mathrm{SR})}\left(E_{\gamma}\right)+f_{M 1}^{(\mathrm{LLR})}\left(E_{\gamma}\right)
$$

Two of these terms, $f_{M 1}^{(\mathrm{SF})}$ and $f_{M 1}^{(\mathrm{SR})}$, are specified in Sec. 3.1.2, while the third one represents an additional, low-lying $M 1$ resonance (LLR) contribution of unknown origin. It is assumed that the energy-smoothed $\gamma$-absorption cross section associated with this term is also of Lorentzian shape. The set of PSFs represented by Eqs. (4.1-4.3), is referred to as "empirical set No. 1", and is shown in Fig. 6. We also performed simulations with the assumption that the LLR term is missing in the expressions for the $M 1 \mathrm{PSF}$, but present in the expression for the $E 1$ or $E 2 \mathrm{PSF}$. In such cases the energies and damping widths of these LLRs were fixed at the values used for the M1 LLR.

A set of selected wide-bin TSC spectra that were obtained from simulations based on the PSFs according to Eqs. (4.1) and (4.3), respectively, are plotted in the right panel of Fig. 4. While simulating these spectra the $E 2 \mathrm{PSF}$ was assumed to have a constant value, $f_{E 2}^{(\mathrm{SP})} \equiv 1.2 \times 10^{-11} \mathrm{MeV}^{-5}$. The agreement between the data and simulation is fairly good. 

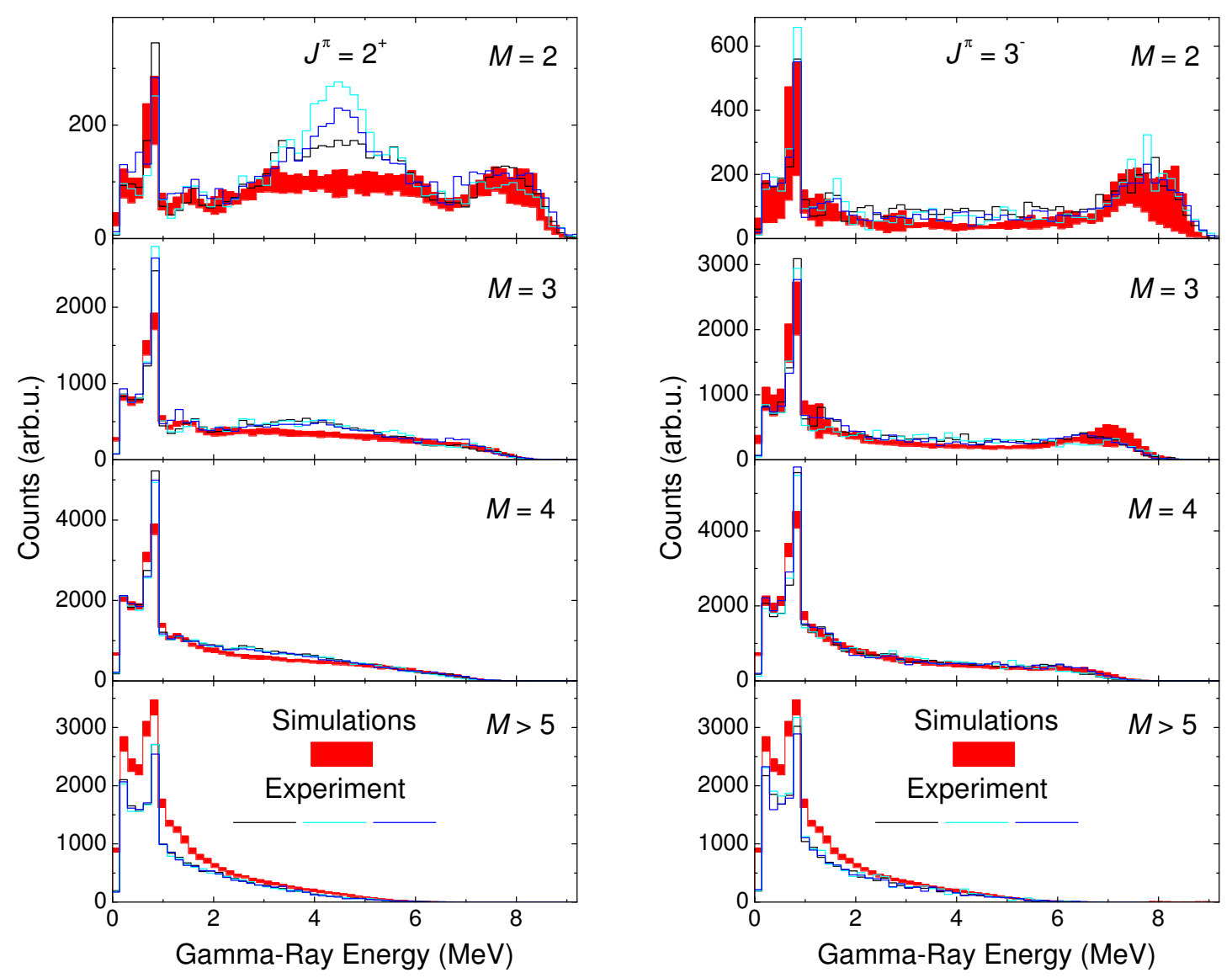

Figure 5: Comparison of experimental and simulated singles spectra for different multiplicities from the decay of $2^{+}$and $3^{-}$resonances. The vertical range of the color area is equal to two rms values deduced from behavior of nuclear realizations.

In addition to $f^{(\text {emp.1) }}$ we have also found another model, referred to as "empirical set No. 2" that gives a very good description of the experimental data:

$$
f_{E 1}^{(\mathrm{emp} .2)}\left(E_{\gamma}, T\right)=f_{E 1}^{(\mathrm{GLO})}\left(E_{\gamma}, T\right), \quad f_{M 1}^{(\mathrm{emp} .2)}\left(E_{\gamma}\right)=f_{M 1}^{(\mathrm{SF})}\left(E_{\gamma}\right)+f_{M 1}^{(\mathrm{SP})}, \quad f_{E 2}^{(\mathrm{emp} .2)}=f_{E 2}^{(\mathrm{SP})}
$$

The most important feature of this set is that none of the PSFs contains a LLR enhancement term. In the expression given by Eq. (4.4) we took $f_{M 1}^{(\mathrm{SP})}=1 \times 10^{-9} \mathrm{MeV}^{-3}, E_{M 1}^{(\mathrm{SF})}=8.95 \mathrm{MeV}$, $\Gamma_{M 1}^{(\mathrm{SF})}=4 \mathrm{MeV}, \sigma_{M 1}^{(\mathrm{SF})}=0.4 \mathrm{mb}$, and $f_{E 2}^{(\mathrm{SP})}=1.2 \times 10^{-11} \mathrm{MeV}^{-5}$.

Singles spectra for different multiplicities from resonance neutron capture obtained with the model $f^{\text {(emp.1) }}$ are shown in Fig. 7. The agreement between the data and simulations is almost ideal. The model $f^{(\text {emp.1) }}$ is evidently much closer to reality than models with a strong enhancement of PSFs at low $E_{\gamma}$ 's. Simulated spectra from resonances obtained with $f^{(\mathrm{emp} .2)}$ are very similar to those shown in Fig. 7; it is difficult to decide which of the empirical models is better.

It should also be pointed out that, independently of the model of PSFs used, the predicted TSC intensities are insensitive to details of the parity dependence of the level density. In other words, the same TSC intensities are expected with a level density that is completely parity-independent above $E_{\text {crit }}$, as with that given by Eq. (3.8). This is not the case for singles spectra following the 

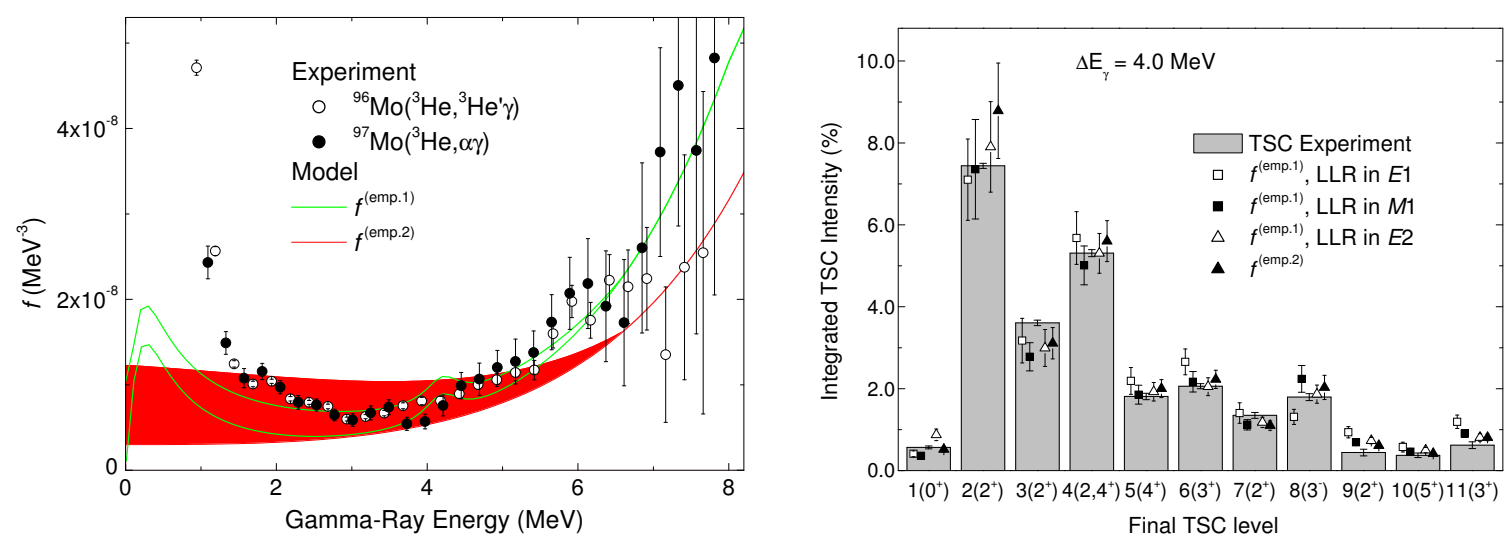

Figure 6: The same as in Fig. 3 but for models of PSFs that give a good description of integrated TSC intensities.

resonance neutron capture. Spectra from $J=2^{+}$for $M=2$ are especially sensitive to the parity dependence of the level density. In fact, parameters $\Delta_{\pi}$, and $C_{\pi}$, see Sec. 3.2 were determined with the help of these spectra.

\section{Discussion and Conclusions}

The results from two $(\mathrm{n}, \gamma)$ experiments on ${ }^{95}$ Mo target - namely from study of TSCs following the thermal neutron capture and $\gamma$-ray spectra following the capture on isolated resonances - are reported. Predictions of the integrated and wide-bin TSC intensities as well as singles spectra for individual multiplicities from resonance capture based on the mixed PSF deduced from the data on the $\left({ }^{3} \mathrm{He},{ }^{3} \mathrm{He}^{\prime} \gamma\right)$ and $\left({ }^{3} \mathrm{He}, \alpha \gamma\right)$ reactions [2] are in sharp disagreement with the results from $(\mathrm{n}, \gamma)$ experiments. The disagreement is mainly due to the large softpole term of the mixed PSF seen from ${ }^{3} \mathrm{He}$-induced reactions.

With the aid of the trial-and-error approach applied to the observables reported in this paper, the shapes and sizes of the $E 1$ and $M 1$ PSFs were estimated. The PSFs obtained are compatible with the existence of a low-lying Lorentzian resonance of unknown multipolarity at $\gamma$-ray energy near $1 \mathrm{MeV}$. However, the strength carried by this resonance is approximately by a factor 5 smaller than that carried by the softpole term. In addition, equally good fits were obtained with PSFs that did not include any enhancement at low energies. Before reaching a firm conclusion regarding a possible low-energy enhancement of the photon strength, further experimental data are needed.

The difference in shapes of the PSFs deduced from ${ }^{3} \mathrm{He}$-induced and $(\mathrm{n}, \gamma)$ reactions might be explained in many ways. For example:

- The softpole is an artifact of the rather complicated method used for processing the data from ${ }^{3} \mathrm{He}$-induced reactions. Thorough validation tests of the method would be valuable. To shed more light on this problem such testing is under way.

- The decay of initial states populated from ${ }^{3} \mathrm{He}$-induced reactions may not be accurately described by pure compound nuclear decay - for example the system may not be completely thermalized before decaying. Then the assumptions of the statistical model could not be reliably applied. 

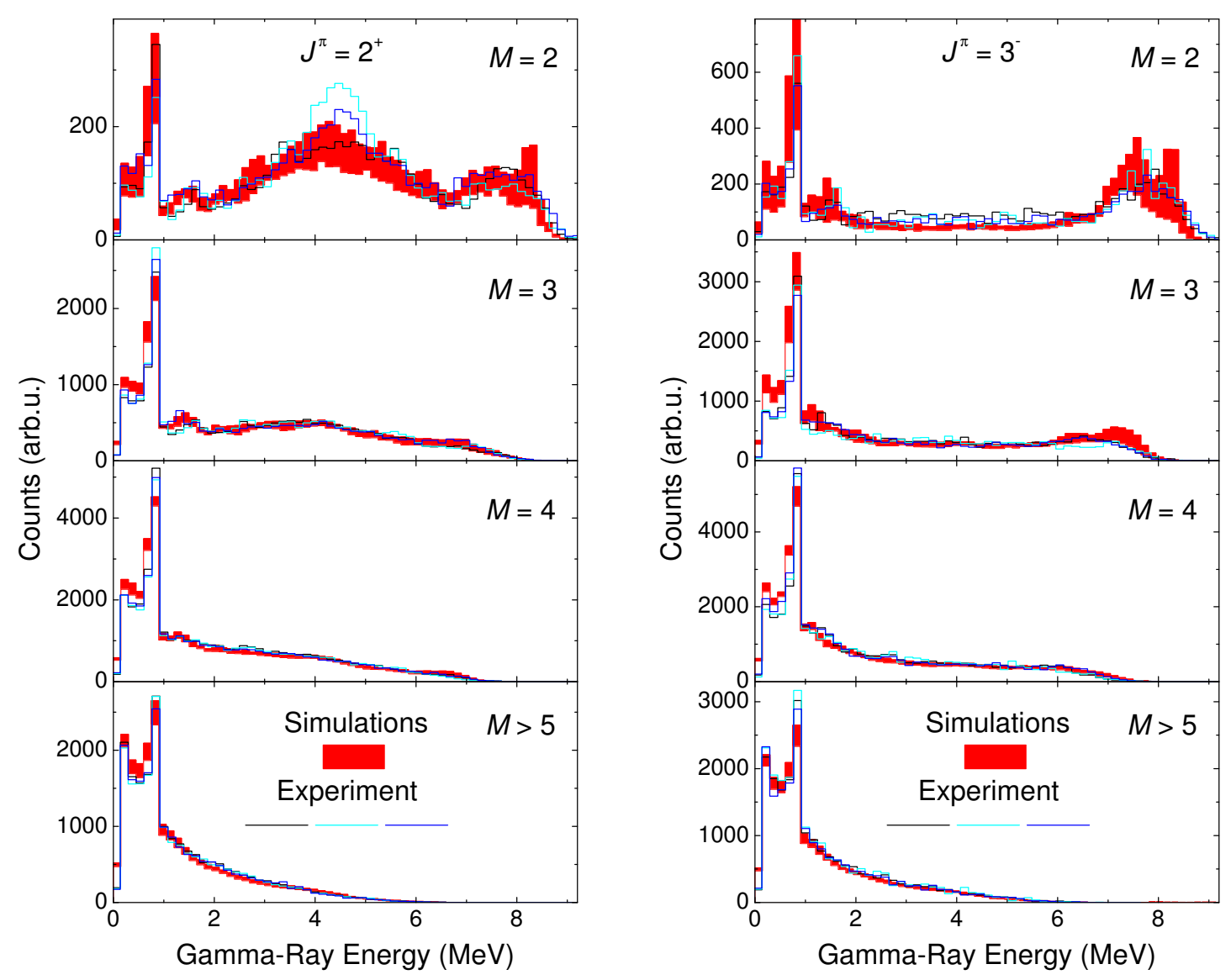

Figure 7: The same as in Fig. 4 but for PSF models given by Eqs. (4.1) and (4.3).

- Although the spins of initial states in ${ }^{3} \mathrm{He}$-induced and $(\mathrm{n}, \gamma)$ reactions are not very different, they are not the same. Initial spins in the (n, $\gamma$ ) reactions studied are mainly $J=2,3$, while those in the ${ }^{3} \mathrm{He}$-induced reactions are probably $J=2-6$. Such a difference could lead, at least in principle, to a difference in the $\gamma$-decay. However, this seems unlikely, since there is no evidence for a strong $J$-dependence of PSFs.

\section{Acknowledgments}

This work was supported in part by the U. S. Department of Energy Grants No. DE-FG5206NA26194, No. DE-FG02-97-ER41042 and No. DE-AC52-06NA25396, and was performed under the auspices of the U. S. Department of Energy by the University of California, Lawrence Livermore National Laboratory and Los Alamos National Laboratory under contract Nos. W7405-ENG-48 and W-7405-ENG-36, respectively. This work has benefited from the use of the LANSCE accelerator facility, supported under DOE contract No. W-7405-ENG-36 and it was also partly supported by the research plan MSM 0021620859 of the Ministry of Education of the Czech Republic. 


\section{References}

[1] A. Voinov et al., Phys. Rev. Lett. 93, 142504 (2004).

[2] M. Guttormsen et al., Phys. Rev. C 71, 044307 (2005).

[3] A. Voinov et al., Phys. Rev. C 74, 014314 (2006).

[4] D.M. Brink, Ph.D. Thesis, Oxford University, 1955.

[5] F. Bečvář et al., Nucl. Instr. and Meth. B 930, (2007).

[6] M. Krtička, private communication.

[7] J. Honzátko et al., Nucl. Instr. and Meth. A376, 434 (1996).

[8] L. Zanini et al., Phys. Rev. C 68, 014320 (2003).

[9] Handbook of Prompt Gamma Activation Analysis with Neutron Beams, edited by G.L. Molnár (Kluwer Academic Publishers, Boston 2004); T. Belgya, private communication.

[10] C.E. Porter and R.G. Thomas, Phys. Rev. 104, 483 (1956).

[11] P.W. Lisowski et al., Nucl. Sci. Eng. 106, 208 (1990).

[12] R. Reifarth et al., Nucl. Instr. Meth. Phys. Res. A531, 530 (2004).

[13] J.M. Wouters et al., IEEE Transactions on Nuclear Science 53, 880 (2006).

[14] G.E. Mitchell et al., Contribution to this workshop.

[15] F. Bečvář, Nucl. Instr. Meth. A417, 434 (1998).

[16] L.K. Peker, Nuclear Data Sheets 68,165 (1993)

[17] P. Axel, Phys. Rev. 126, 671 (1962).

[18] S.G. Kadmenskij, V.P. Markushev and V.I. Furman, Sov. J. Nucl. Phys. 37, 165 (1983).

[19] J. Kopecky and M. Uhl, Phys. Rev. C 41, 1941 (1990), and references therein.

[20] G.A. Bartholomew, Ann. Rev. of Nucl. Sci. 11, 259, (1961).

[21] J. Kopecky, Handbook for calculations of nuclear reaction data, IAEA-TECDOC-1034, August 1998, p.97, available at http://iaeand.iaea.or.at/ripl

[22] S.S. Dietrich and B.L. Berman, At. Data and Nucl. Data Tables 38, 199 (1988).

[23] L.M. Bollinger, in Experimental Neutron Resonance Spectroscopy, edited by J.A. Harvey (Academic, New York, 1970).

[24] C. Fransen et al., Phys. Rev. C 67, 024307 (2003).

[25] C. Fransen et al., Phys. Rev. C 70, 044317 (2004).

[26] T. von Egidy, H.H. Schmidt and A.N. Behkami, Nucl. Phys. A481, 189 (1988).

[27] S.I. Al-Quraishi et al., Phys. Rev. C 67, 015803 (2003).

[28] R. Chankova et al., Phys. Rev. C 73, 034311 (2006). 